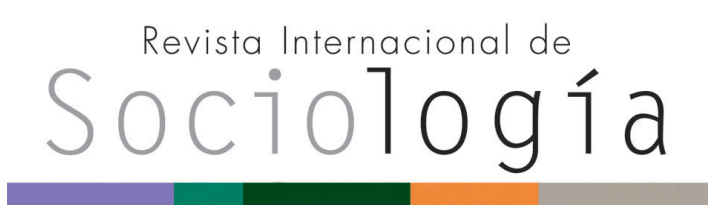

Revista Internacional de Sociología RIS vol. 73 (3), e018, septiembre-diciembre, 2015, ISSN-L:0034-9712

DOI: http://dx.doi.org/10.3989/ris.2015.73.3.e018

\section{CONOCIMIENTO, VALORES E INTENCIONES COMO DETERMINANTES DEL COMPORTAMIENTO ECOLÓGICO}

\author{
López Miguens, María Jesús \\ Universidad de Vigo, España. \\ chusl@uvigo.es \\ Álvarez González, Paula \\ Universidad de Vigo, España. \\ paula_ag@uvigo.es \\ GonZÁlez VÁzQuez, EnCARnación \\ Universidad de Vigo, España. \\ egzlez@uvigo.es
}

Como citar este artículo / Citation: López Miguens, M. J., Álvarez González, P. y González Vázquez, E. 2015. «Conocimiento, valores e intenciones como determinantes del comportamiento ecológico». Revista Internacional de Sociología, 73. 3 DOl: http://dx.doi.org/10.3989/ris.2015.73.3.e018

\footnotetext{
REsUmen

El objetivo de este trabajo es explicar el comportamiento ecológico de los individuos. En particular, se analiza el papel que, sobre el comportamiento, ejercen el conocimiento sobre el medio ambiente, los valores de las personas y sus intenciones a través de la propuesta de un modelo. Con carácter previo se han validado las escalas de medida específicas de cada constructo. La técnica estadística utilizada es la modelización de ecuaciones estructurales aplicada sobre una muestra de 497 individuos. El modelo testado es capaz de explicar el $87 \%$ de la varianza del comportamiento ecológico. Nuestros resultados indican que el conocimiento y los valores son susceptibles de generar de forma indirecta actuaciones ecológicas por parte del individuo, mediadas por las intenciones; mientras que las intenciones ejercen una influencia directa sobre la conducta.
}

\section{KNOWLEGDE, VALUES AND INTENTIONS AS DETERMINANTS OF THE ECOLOGICAL BEHAVIOR}

Copyright: @ 2015 CSIC. Este artículo de acceso abierto distribuido bajo los términos de la licencia Creative Commons Attribution-Non Commercial (by-cn) Spain 3.0. Recibido: 14/01/2014. Aceptado: 29/09/2014

Publicación online: 29/10/2015

\section{AbSTRACT}

The aim of this paper is to explain the ecological behavior of individuals. In particular, the role that knowledge about the environment, values and intentions played in the realization of an ecological behavior has been analyzed. Previously, the measurement scales of each construct have been validated. A structural equation modeling was used to a sample of 497 individuals. The tested model explained 87 per cent of the variance of ecological behavior. Our results show that knowledge and values are able to generate, indirectly, environmental actions, mediated by intentions, while intentions have a direct influence on behavior.

\section{KEYWORDS}

Attitudes; Scales; Structural Model.

\section{Palabras Clave}

Actitudes; Escalas; Modelo estructural. 


\section{INTRODUCCIÓN}

En los últimos años, los problemas ambientales, como el cambio climático, la contaminación atmosférica y del agua, la destrucción de los recursos naturales o la utilización de elementos químicos en los productos de uso diario, han propiciado la evolución de las preocupaciones y los valores de la sociedad hacia aquellos relacionados con la protección, la defensa del medio ambiente y su preservación para generaciones futuras (Akehurst, Afonso y Gonçalves 2012; Eurobarómetro 2011; Ministerio de Medio Ambiente, Medio Rural y Marino de España 2011); es decir, hacia la sostenibilidad medioambiental. A su vez, esto ha motivado un cambio en el comportamiento y los hábitos de vida y consumo de los individuos (Young, Hwang, Mcdonald y Oates 2010; Schiffman, Kanuk y Hansen 2008; Vozmediano y San Juan 2005; Vicente y Aldamiz-Echevarría 2003).

La modificación de los comportamientos y hábitos de vida y consumo hacia otros más ecológicos, que pretenden la defensa y protección del medio ambiente, ha suscitado el interés de los investigadores (Luzio y Lemke 2013; Do Paço y Raposo 2009; Calvo, Aguilar y Berrios 2008) que, en primer lugar, buscan entender qué aspectos o variables hacen que un individuo se comporte de forma ecológica y, en segundo lugar, analizar los instrumentos que permitan medir esas variables.

Los trabajos previos que estudian las razones por las que las personas adoptan un comportamiento ecológico no son concluyentes. Por un lado, nos encontramos investigaciones que utilizan una única variable para su predicción, como la preocupación medioambiental (Vozmediano y San Juan 2005; Roberts y Bacon 1997) o los valores y creencias (Calvo et al. 2008; Fraj y Martínez 2004; González y Amérigo 1999). No obstante, estos mismos trabajos sugieren que la utilización de una única variable es válida pero no suficiente para predecir comportamientos ecológicos. Por otro lado $y$, al mismo tiempo, han surgido otras investigaciones que plantean modelos más completos, incluyendo más variables, como el conocimiento sobre el medio ambiente, los valores del individuo o sus intenciones (Aguilar-Luzón, García-Martínez, Calvo-Salgueiro y Salinas 2012; Jiménez y Lafuente 2010; Bamberg y Möser 2007; Fraj y Martínez 2006; López y García 2007; Kaiser, Wölfing y Fuhrer 1999), y que han conducido a la obtención de predictores más consistentes. Sin embargo, hasta donde hemos podido saber, estos estudios no logran explicar más allá de un $40 \%$ del comportamiento ecológico de una persona.

Además, tal y como refleja Amérigo (2006) no se observa en la literatura un consenso sobre cuáles son las variables que mayor influencia tienen en la realización de un comportamiento ecológico.

En cuanto a su medición, la ausencia de una definición precisa y consensuada de cada constructo (op. cit.) ha fomentado la proliferación de numerosas escalas de medición que han sido aplicadas en las diferentes investigaciones, lo que dificulta la comparación de resultados entre estudios. En España, la investigación sobre escalas de actitudes ambientales y comportamiento ecológico presenta varias particularidades. En un análisis de contenido realizado sobre 27 artículos publicados por investigadores españoles en los últimos años, Amérigo (ibid.) pone de manifiesto la inexistencia de generalización en el empleo de escalas de medida ya que estas son empleadas únicamente en el proyecto en el que han sido desarrolladas, las investigaciones se basan en muestras universitarias y de estudiantes (Barreiro, López, Losada y Ruzo 2002), en una única región (González 2002) y a menudo las investigaciones se realizan a nivel exploratorio (p. ej., Vozmediano y San Juan 2005). Todo ello suscita un especial interés por la validación de las escalas a nivel confirmatorio, utilizando una muestra representativa de la población general, para, de este modo, proporcionar unas escalas de medida válidas y fiables que puedan ser utilizadas en los estudios sobre comportamiento ecológico y sus antecedentes.

El presente trabajo de investigación contribuye al estudio en el ámbito del comportamiento ecológico de un individuo al: 1) plantear un modelo estructural provisto de factores de índole cognitivo, afectivo y conativo (conocimiento, valores e intenciones), que permita un mejor entendimiento del comportamiento ecológico de un individuo y; 2) validar escalas de medida ya existentes para estas variables, adaptándolas al contexto español y empleando para ello una muestra extraída de la población general.

Con este propósito, el trabajo se estructura como sigue. En la siguiente sección se efectúa una revisión de la literatura relevante, se formulan las hipótesis causales y se propone el modelo a contrastar en el análisis empírico. A continuación se describe la metodología utilizada, seguida de los resultados obtenidos, sobre los que emitiremos las conclusiones y las recomendaciones estratégicas. Por último, se señalan las principales limitaciones y orientaciones para futuras investigaciones.

\section{EL COMPORTAMIENTO ECOLÓGICO DE LOS INDIVIDUOS: DETERMINANTES Y PROPUESTA DE UN MODELO}

El comportamiento ecológico de un individuo puede definirse como la realización de acciones que pretenden preservar o conservar 0 , al menos, no perjudicar al medio ambiente (Kaiser et al. 1999). Estas acciones pueden ser muy variadas y comprenden la modificación de los hábitos de vida y de consumo del individuo, adquiriendo productos más ecológicos y respetuosos con el medio ambiente 0 
dejando de adquirir productos contaminantes, mostrándose favorable a un modelo de crecimiento económico racional (o sostenible), la utilización de los productos adquiridos de una forma ecológica o la concienciación de la necesidad de reutilizar o reciclar los residuos, utilizando medios de transporte más ecológicos, participando de forma activa en manifestaciones y grupos ambientalistas, ahorrando energía y agua, etc. (Izagirre-Olaizola, Fernández-Sainz y Vicente-Molina 2013; Do Paço y Raposo 2009), con el objetivo de dar respuesta a los problemas ambientales que les preocupan (Fraj, Martínez y Grande 2004; Vicente y Aldamiz-Echevarría 2003).

Para entender el comportamiento ecológico de un individuo, en la literatura se han utilizado distintas variables (Esteban, Mondéjar y Cordente 2009): 1) variables demográficas y socioeconómicas como género, edad, nivel de estudios, clase social, status, renta, ideología política, etc. 0 2) variables psicográficas y de conocimiento, que incluyen los valores, estilos de vida, las características de la personalidad, las actitudes y la cantidad de información y conocimiento sobre problemas ambientales. No obstante, autores como Peattie (2001), Fraj, Grande y Martínez (2002) o Fraj y Martínez (2006) encuentran que en la literatura las variables sociodemográficas han sido consideradas poco relevantes o significativas para explicar el comportamiento, siendo las variables psicográficas y de conocimiento las que reciben una mayor atención. En consecuencia, el presente estudio opta por el análisis de estas últimas y, particularmente, por los componentes de la actitud (Kaiser et al., 1999). Siguiendo la teoría de la acción razonada (Fishbein y Ajzen 1975) y su extensión, la teoría de la acción planificada (Ajzen 1991), una actitud se define como «el sentimiento positivo o negativo que un individuo tiene sobre un objeto, un aspecto del mundo físico, una persona, un concepto abstracto o un comportamiento determinado» y se compone de: lo que piensa, lo que siente y su predisposición a manifestar lo que piensa y siente. De este modo, para los propósitos de esta investigación la actitud ambiental o hacia el medio ambiente es entendida como el conjunto de factores psicológicos específicos relacionados con la propensión de los individuos para llevar a cabo comportamientos proambientales o ecológicos (Aguilar-Luzón et al. 2012; Jiménez y Lafuente 2010; Bamberg y Möser 2007; Fraj y Martínez 2006; Milfont y Duckitt 2004; Kaiser et al. 1999; Hines, Hungerford y Tomera 1987), formada por tres elementos: 1) el conocimiento sobre los problemas ambientales que una persona ha adquirido a través de su tradición cultural, de la difusión en los medios de comunicación de conocimientos científicos y de su propia experiencia (Frick, Kaiser y Wilson 2004; Barreiro, López, Losada y Ruzo 2002), cuestiones que le permiten configurar lo que piensa sobre el medio ambiente; 2) los sentimientos que genera el medio ambiente en base a los valores y creencias del propio individuo y que guiarán sus acciones (González y Amérigo 1998; Stern y Dietz 1994; Stern, Dietz y Kalof 1993); y, por último 3) la intención o disposición a realizar la acción de comportarse de forma ecológica (Castro 2002). Los tres componentes de la actitud, esto es, el conocimiento, los valores y la intención de comportamiento, se conforman como los principales antecedentes del comportamiento ecológico y, de un modo similar a como lo han presentado Kaiser et al. (íbid.) en su trabajo, serán utilizados en nuestro modelo de un modo secuencial para predecir el comportamiento ecológico.

Los estudios previos que han analizado la relación entre la actitud y el comportamiento ecológico no muestran resultados concluyentes, y a menudo han puesto de manifiesto la débil utilidad de la actitud como predictor de la conducta ecológica (Moreno, Corraliza y Ruiz 2005; Hines et al. 1987), posiblemente debido a las diferencias en el nivel de especificidad de las medidas utilizadas (Kaiser et al. 1999; Roberts y Bacon 1997). Para evitar los problemas de incongruencia de resultados, ambas variables deben medirse al mismo nivel de especificidad, ya sea general o específico. No obstante, autores como Kaiser et al. (íbid.) defienden que las medidas generales son ocasionalmente preferidas a las específicas, ya que estas últimas son más susceptibles a influencias del entorno y que se encuentran fuera del control de los individuos. Siguiendo esta postura de investigación se utilizan medidas generales de los componentes de la actitud medioambiental y del comportamiento ecológico.

En la literatura, el conocimiento es comúnmente visto como una condición previa necesaria, aunque no suficiente, para el comportamiento de una persona (Fraj et al. 2004; Kaiser y Fuhrer 2003) y ha sido utilizado en diversos estudios sobre el comportamiento ecológico de un individuo (p. ej. Brosdahl y Carpenter 2010; Fraj y Martínez 2005; Barreiro et al. 2002; Kaiser et al. 1999). En estas investigaciones los autores confirman el poder predictivo del conocimiento aunque concluyen que, por sí solo, este conocimiento, no se traduce en un comportamiento, sino que su efecto es indirecto (Bamberg y Möser 2007). En consecuencia, se sugiere que existen variables que median la relación entre el conocimiento y el comportamiento ecológico, tales como las intenciones (Kaiser et al. Íbid.). Con base en los estudios anteriores, se propone:

- Hipótesis 1: El conocimiento está positivamente relacionado con la realización de comportamientos ecológicos mediado por la variable intención de comportamiento.

En cuanto a los valores, autores como Stern et al. (1993) sugieren que los individuos combinan sus valores y creencias para construir las actitudes que 
guiarán sus acciones. Entienden, por lo tanto, que «los valores actúan como un filtro que modula la información disponible sobre la situación, el objeto o la conducta y si es congruente con los valores del individuo, esta persona desarrollará unas creencias más positivas hacia dicha situación y por tanto, facilitarán la realización de la conducta» (Aguilar, Monteoliva y García 2005: 25). Varios estudios han demostrado la relación entre los valores y el comportamiento ecológico (Fraj y Martínez 2004; Stern, Dietz, Kalof y Guagnano 1995). No obstante, la relación aumenta si se considera la intención de comportamiento como variable mediadora (Kaiser et al. 1999). Esto lleva a plantear la siguiente hipótesis:

- Hipótesis 2: Los valores están positivamente relacionados con la realización de comportamientos ecológicos mediados por la variable intención de comportamiento.

La intención de comportamiento o conductual puede definirse como la 'disposición a realizar cierta clase de acción' y ha sido considerada como el principal predictor (Fishbein y Ajzen 1975) o la variable inmediatamente anterior (Fraj y Martínez 2005; Castro 2002; Hines et al. 1987) a un comportamiento determinado. Esto significa que el carácter deliberado de un comportamiento específico, como puede ser el comportamiento ecológico, implica una intención previa a realizarlo (Castro 2002). De este modo, se propone:

- Hipótesis 3: La intención conductual tiene una influencia positiva y directa en el comportamiento ecológico de un individuo.

Así, utilizando como marco teórico la teoría de la acción razonada, el estudio busca definir las relaciones entre los tres componentes de la actitud ambiental (conocimiento, valores e intenciones) y el comportamiento ecológico (Figura 1).

Figura 1.

Modelo estructural propuesto.

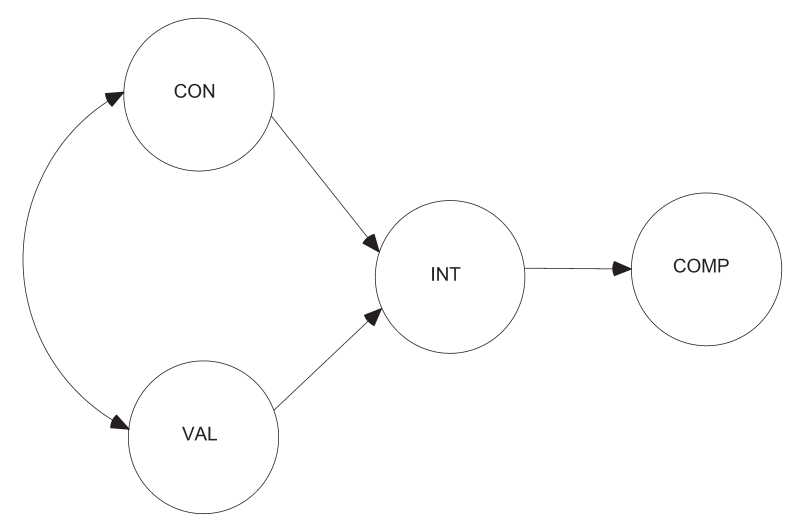

\section{Metodología}

\section{Ficha técnica de la investigación}

Los datos han sido obtenidos a través de un cuestionario autoadministrado durante los meses de febrero a junio de 2011. En ese periodo se han recogido un total de 500 cuestionarios, resultando válidos, tras la depuración de los datos, 497. El procedimiento de muestreo seguido ha sido por cuotas de acuerdo a la edad y género de la pirámide de población española (Instituto Nacional de Estadística 2011).

El análisis de los datos se ha realizado en dos etapas. En primer lugar, se ha evaluado el cumplimiento de las propiedades psicométricas de las escalas reflexivas empleadas mediante el análisis factorial a nivel exploratorio, inicialmente, y confirmatorio (CFA) después. A continuación, se comprobó el ajuste del modelo propuesto y las hipótesis de investigación a través del análisis de ecuaciones estructurales (SEM). Con el fin de evadir la ausencia de normalidad multivariante y puesto que el tamaño muestral supera los 250 elementos (Willett y Sayer 1994), se ha utilizado, para la estimación del modelo el método asintóticamente libre de distribución, actuando directamente con los datos sin necesidad de remuestreo (bootstrapping).

\section{Medidas}

Para medir las variables incluidas en el modelo se han utilizado diferentes instrumentos, basados en escalas tipo Likert de siete puntos, que van desde 1 (totalmente en desacuerdo) a 7 (totalmente de acuerdo). La variable conocimiento medioambiental fue medida a través de la escala propuesta por Kaiser et al. (1999). Esta escala incluye 10 ítems sobre el conocimiento general que un individuo tiene sobre el medio ambiente y los problemas medioambientales, sobre las causas de los problemas ecológicos y sus diferentes efectos y consecuencias. Los valores se miden mediante la escala utilizada en la investigación de González (2002) y está compuesta por 17 ítems, 15 de ellos extraídos del trabajo de Schwartz (1992), relacionados con el universalismo, el poder y el logro o la estimulación, y 2 empleados por Stern y Dietz (1994), relacionados con el medio ambiente y su protección. La intención para realizar comportamientos ecológicos por parte de un individuo, que suponen ciertos esfuerzos y/o costes como utilizar un medio de transporte menos contaminante, pagar algún impuesto medioambiental 0 apoyar iniciativas que tengan como objetivo proteger el medio ambiente, se ha medido a través de una escala de 11 ítems, adaptada de la estructura propuesta por Kaiser et al. (ibid.). Y para el comportamiento ecológico, utilizamos la escala de Kaiser (1998), adaptada al contexto de aplicación. Esta escala incluye diversas acciones sobre la participación activa o pasiva del individuo en actividades de conservación del medio ambiente, su actitud hacia el reciclaje, hacia el ahorro de agua y energía, 
hacia la compra de productos menos contaminantes, su compromiso con asociaciones medioambientales y el uso del transporte público para minimizar los impactos ambientales derivados del uso del automóvil. Para este estudio se omitieron los ítems 'suelo comprar leche en botellas retornables', por no ser de aplicación en el contexto español, ya que la mayor parte de la venta de leche en España se realiza en envases de cartón tetrabrik y plástico y no en envases retornables (Del Val 2011) y 'no sé si puedo usar gasolina con plomo en mi coche', por tratarse de un indicador de conocimiento y no de comportamiento. En total se propone la escala compuesta por 28 ítems.

\section{Resultados}

\section{Muestra}

La muestra está compuesta por 246 hombres (49,5\%) y 251 mujeres $(50,5 \%)$ de edades comprendidas, en su mayoría, entre los 16 y 54 años (74,8 \%). En cuanto a su educación, el 64,2 \% poseen estudios secundarios o superiores y manifiestan una orientación política de izquierdas (centro-izquierda e izquierda) en el 31,6\% de los casos frente al 17,6\% de derechas (centro-derecha y derecha). Destacar, asimismo, que el 43,3\% de la muestra apuntan la ausencia de ideología política. Los datos descriptivos de la muestra se resumen en la Tabla 1.

Tabla 1.

Perfil sociodemográfico de la muestra.

\begin{tabular}{lcc}
\hline Variable sociodemográfica & $\begin{array}{c}\text { Frecuencia } \\
(\mathbf{n}: \mathbf{4 9 7})\end{array}$ & $\begin{array}{c}\text { Porcentaje } \\
(\%)\end{array}$ \\
\hline Género & & \\
Mujer & 251 & 50,5 \\
Hombre & 246 & 49,5 \\
\hline Edad & & \\
$16-24$ & 71 & 14,3 \\
$25-34$ & 104 & 20,9 \\
$35-44$ & 100 & 20,1 \\
$45-54$ & 97 & 19,5 \\
$55-64$ & 61 & 12,3 \\
65-74 & 64 & 12,9 \\
\hline Nivel de estudios & & \\
Sin estudios & 55 & 11,1 \\
Estudios primarios & 121 & 24,3 \\
Estudios secundarios & 169 & 34,0 \\
Estudios universitarios & 130 & 26,2 \\
Máster/Doctor & 20 & 4,0 \\
\hline Ideología política & & \\
Derecha & 42 & 8,5 \\
Centro-derecha & 45 & 9,1 \\
Centro & 36 & 7,2 \\
Centro-izquierda & 68 & 13,7 \\
Izquierda & 89 & 17,9 \\
Ninguna & 215 & 43,3 \\
\hline
\end{tabular}

\section{Desarrollo de los instrumentos de medida}

Las escalas de medida propuestas, todas ellas de naturaleza reflexiva, fueron evaluadas siguiendo las recomendaciones de Anderson y Gerbing (1988) y de Churchill (1979);es decir, analizando el cumplimiento de sus propiedades psicométricas: validez de contenido, dimensionalidad, fiabilidad y validez convergente $y$ discriminante.

Se garantiza la validación de contenido a través de la revisión de la literatura y de un grupo de expertos. Como consecuencia de este proceso, se han reformulado y adaptado algunos de los ítems que representan el dominio conceptual de las escalas.

Para evaluar la dimensionalidad se ha realizado, a nivel exploratorio, un análisis de componentes principales con rotación Varimax como método de extracción. A continuación se analiza el valor de las comunalidades, lo que permite justificar la eliminación de algunos indicadores. Posteriormente, aplicamos un CFA sobre las escalas teniendo en cuenta los diferentes niveles y evaluamos las medidas de ajuste, los parámetros del modelo propuesto, los valores de la matriz de covarianzas y la correlación múltiple al cuadrado (SMC). Los modelos iniciales arrojan valores por debajo de los niveles aceptables, por lo que se procede a la reespecificación. Los modelos finales de primer y segundo orden resultantes arrojan muy buenos ajustes, como se muestra en la Tabla 2. En consecuencia, se deduce un carácter multidimensional y multinivel para los constructos conocimiento y comportamiento ecológico y unidimensional para las escalas valores e intenciones.

La prueba de fiabilidad se ha efectuado a través de 3 medidas (Tabla 2): 1) los coeficientes Alfa de Cronbach; 2) la correlación ítem total; y 3) la fiabilidad compuesta (FC). Todos los coeficientes Alfa de Cronbach superan o se aproximan al nivel mínimo establecido en 0,70 (Numally 1978), no siendo posible mejorar el Alfa con la eliminación de ningún ítem. Todas las correlaciones ítem-total superan o se acercan al valor de 0,50, y respecto a la FC puede observarse que los valores de los estadísticos superan en todos los casos el valor mínimo recomendado, establecido en 0,7 por Luque (2000), entre otros. Los resultados obtenidos indican, por tanto, una elevada fiabilidad o consistencia interna de las escalas.

La validez convergente se demuestra a través de 2 pruebas (Tabla 2): 1) todas las cargas factoriales de todos los ítems que componen cada medida son significativas; y 2) el valor de la varianza media extraída (AVE) supera el valor de 0,5 (Bagozzi, Yi y Phillips 1991; Fornell y Larcker 1981). 
Tabla 2.

Dimensionalidad, fiabilidad y validez convergente de las escalas de conocimiento, valores, intenciones y comportamiento.

\begin{tabular}{|c|c|c|c|c|c|c|}
\hline $\begin{array}{c}\text { Constructo } \\
\text { Dimensiones } \\
\text { İtems }\end{array}$ & $\begin{array}{l}\text { Cargas } \\
\text { estand. } \\
\left({ }^{*}\right)\end{array}$ & $\begin{array}{l}\text { Alfa de } \\
\text { Cronbach }\end{array}$ & $\begin{array}{c}\text { Posib. } \\
\text { mejora } \\
\text { Alfa }\end{array}$ & Ítem-Total & F.C. & V.E. \\
\hline Conocimiento & & .761 & No & $(, 474-, 578)$ & ,9458 &, 7774 \\
\hline Conocimiento básico (CB) & & , 722 & No & $(, 505-, 589)$ & 9106 &, 7725 \\
\hline $\begin{array}{l}\text { CON1: La fusión de los casquetes polares puede provocar inundaciones de } \\
\text { costas e islas. }\end{array}$ & ,69 & & & & & \\
\hline $\begin{array}{l}\text { CON2: Combustibles fósiles, por ejemplo gas-oil produce } \mathrm{CO} 2 \text { en la atmósfera } \\
\text { cuando se queman. }\end{array}$ &, 71 & & & & & \\
\hline $\begin{array}{l}\text { CON8: El clima mundial probablemente cambie de forma masiva si el } \mathrm{CO}_{2} \text { sigue } \\
\text { siendo emitido a la atmósfera en cantidades tan grandes como lo es ahora. }\end{array}$ &, 70 & & & & & \\
\hline Conocimiento experto(CEX) & & ,687 & No & $(, 525)$ & ,8789 &, 7842 \\
\hline $\begin{array}{l}\text { CON4: Metales tóxicos se introducen en la cadena alimenticia, por ejemplo, a } \\
\text { través de las aguas subterráneas. }\end{array}$ & ,75 & & & & & \\
\hline CON7: Metales tóxicos permanecen en el cuerpo humano. &, 70 & & & & & \\
\hline \multicolumn{7}{|c|}{$X^{2}=12,259 ;$ gl. $=6 ; p=, 056 ; X^{2}$ normalizada $=2,043 ;$ RMSEA=,046; CFI=,956 } \\
\hline Valores & & ,879 & No & $(, 563-, 764)$ & ,9779 & ,8338 \\
\hline VAL5: Un mundo en paz (Libre de guerras y conflictos). & ,59 & & & & & \\
\hline VAL6: Unión con la naturaleza (Integrarse con la Naturaleza). & 72 & & & & & \\
\hline VAL9: Un mundo bello (Belleza en la Naturaleza y en las artes). & 64 & & & & & \\
\hline VAL10: Justicia social (Corregir injusticias, cuidar de los débiles). & ,67 & & & & & \\
\hline VAL11: Respeto por la tierra (Armonía con otras especies). & ,77 & & & & & \\
\hline VAL14: Proteger el medio ambiente (Conservar la Naturaleza). & ,84 & & & & & \\
\hline VAL17: Evitar la contaminación (Conservar los recursos). &, 75 & & & & & \\
\hline
\end{tabular}

$X^{2}=20,100 ;$ gl. $=14 ; p=, 127 ; X^{2}$ normalizada $=1,436 ;$ RMSEA=,030; $C F I=, 957$

Intención de comportamiento

INT6: Preferiría no tener que conducir para ir a trabajar.

INT7: Preferiría ir de compras sin mi automóvil.
$693 \quad$ No

, 76

, 72
$(, 531) \quad$, 8441 $\quad, 7305$

$\mathrm{X}^{2}=, 000 ; \mathrm{gl} .=0 ; \mathrm{CFl}=1$

Comportamiento ecológico

Gestión ecológica de basuras (GEB)

COMP4: Recojo y reciclo el papel usado.

COMP5: Llevo botellas vacías a un contenedor de reciclaje.

Implicación medioambiental (IM)

COMP23: A menudo hablo con amigos sobre problemas relacionados con el

medio ambiente.

COMP25: En el pasado, he llamado la atención a alguien respecto a su comportamiento anti-ecológico.

$\begin{array}{rrrrrr} & , 721 & \text { No } & (, 428-, 579) & , 930 & , 691 \\ & , 826 & \text { No } & (, 704) & , 892 & , 805 \\ , 70 & & & & & \\ , 92 & & & & & \\ & , 660 & \text { No } & (, 492) & , 838 & , 722 \\ & & & & & \\ , 76 & & & & & \\ & & & & & \end{array}$

$X^{2}=2,470 ;$ gl.=2; $p=, 291 ; X^{2}$ normalizada=1,235; RMSEA=,023; CFI=,999

\footnotetext{
$\left({ }^{*}\right)$ Significativas.

n.a.: No aplicable. Elemento fijado para la identificación del modelo.

F.C.: Fiabilidad compuesta; V.E: Varianza extraída.
}

La validez discriminante fue evaluada a través del desarrollo de tres pruebas (Tabla 3); la primera, de naturaleza exploratoria, y las dos siguientes, confirmatoria: 1) la comprobación de la existencia de bajas correlaciones entre constructos (por debajo de 0,8, según Hair, Anderson, Tatham y Black 1999); 2) demostrar que el valor resultado de aplicar el cuadrado a la correlación entre cada par de factores de un constructo no supera la varianza extraída obtenida para cada factor estudiado (Fornell y Larcker 1981); 3) calcular los intervalos de confianza para la correlación de los constructos y comprobar que ninguno de ellos contenga la unidad (Anderson y Gerbing 1988). Los resultados alcanzados permiten confirmar la existencia de validez discriminante.

\section{Modelo estructural}

Para la validación del modelo causal propuesto se ha utilizado el procedimiento SEM, y en particular las recomendaciones de Levy y Varela (2003), que permiten examinar de forma simultánea las relaciones directas e indirectas que se producen entre los constructos. De este modo, para la evaluación de la calidad del ajuste del modelo 
Tabla 3.

Resumen de las medidas de validez discriminante.

\begin{tabular}{|c|c|c|c|c|}
\hline Constructos & Correlación & $\begin{array}{c}\text { Correlación al } \\
\text { cuadrado }\end{array}$ & Varianza extraída & $\begin{array}{c}\text { Intervalos de } \\
\text { confianza }\end{array}$ \\
\hline Conocimiento-valores &, 517 & ,267 & & $(, 409 ;, 625)$ \\
\hline Conocimiento-intención & ,399 &, 159 & Conocimiento: ,7774 & $(, 235 ;, 563)$ \\
\hline Conocimiento-comportamiento &, 593 &, 351 & Valores: ,8338 & $(, 447 ;, 739)$ \\
\hline Valores-comportamiento &, 568 & ,322 & Intención: ,7305 & $(, 44 ;, 696)$ \\
\hline Valores-intención & ,252 &, 063 & Comportamiento: ,691 & $(, 122 ;, 382)$ \\
\hline Intención-comportamiento & ,661 &, 436 & & $(, 433 ;, 889)$ \\
\hline
\end{tabular}

a los datos, se analiza el indicador SMC (coeficiente de correlación múltiple), las estimaciones infractoras (errores estandarizados), los índices de ajuste y la significación de las relaciones causales. Los resultados iniciales obtenidos apoyan la reespecificación del modelo inicial propuesto, sugiriendo la eliminación de los ítems VAL5 y VAL17 y dando lugar al modelo revisado que se muestra en la Figura 2.

Figura 2.

Modelo estructural final.

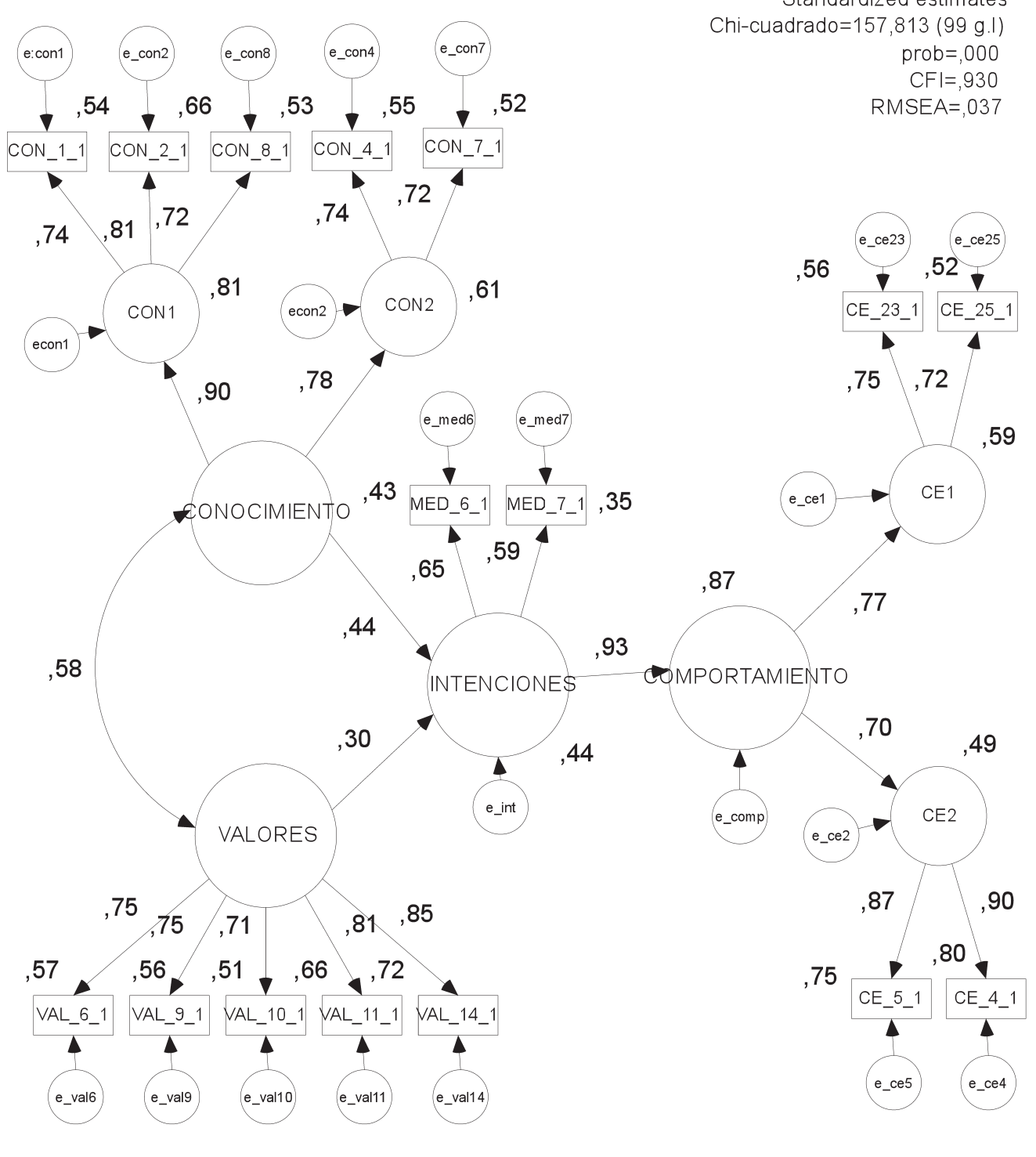


Tabla 4.

Medidas de ajuste del modelo final y contrastación de hipótesis.

\begin{tabular}{|c|c|c|c|c|c|}
\hline \multicolumn{6}{|c|}{ Índices de ajuste del modelo } \\
\hline Chi-Cuadrado & & & & $15 /, 813$ & \\
\hline Chi-Cuadrado Normalizada & & & & 1,594 & \\
\hline GFI & & & & ,951 & \\
\hline AGFI & & & & ,933 & \\
\hline CFI & & & & ,930 & \\
\hline RMSEA & & & & 037 & \\
\hline Relación causal & Signo esperado & $\begin{array}{c}\text { Carga } \\
\text { Estandarizada }\end{array}$ & C.R & S.E. & Test \\
\hline $\mathrm{H} 1: \mathrm{CON} \rightarrow \mathrm{INT}$ & + &, 440 & 5,310 & 097 & Aceptada \\
\hline $\mathrm{H} 2:$ VAL $\rightarrow$ INT & + & 303 & 4,455 & 078 & Aceptada \\
\hline H3: INT $\rightarrow$ COMP & + & ,932 & 9,498 & ,104 & Aceptada \\
\hline
\end{tabular}

n.a.: No aplicable. Elemento fijado para la identificación del modelo.

El coeficiente SMC de determinación o de fiabilidad para este modelo estructural final refleja valores suficientes (por encima o muy próximos a 0,50 ) para todos los constructos de los diferentes niveles. Sus valores fluctúan desde 0,44 para el constructo intenciones hasta 0,87 para el comportamiento ecológico, con lo que se asegura la representatividad de todas las variables en el modelo. Se destaca el porcentaje de la varianza explicada del constructo comportamiento ecológico por el modelo, que alcanza el $87 \%$. Respecto a la bondad de ajuste, el modelo arrojó un valor de Chi-cuadrado de 157,813 con 99 grados de libertad $(p=0,000)$, como se presenta en la Tabla 4. Se trata de un test muy recomendable para tamaños de muestra de 100-200 elementos (Levy y Varela 2003; Hu y Bentler 1999; Bollen 1989). Sin embargo, si la muestra es de tamaño superior (como es nuestro caso, que contabiliza un total de 497), el test aconseja el rechazo de la hipótesis nula incluso con diferencias sustancialmente no significativas, aunque estadísticamente lo sean. De este modo, se utilizaron, para la evaluación del ajuste del modelo, los índices Chi-cuadrado normalizada, GFI, AGFI, CFI y RMSEA (Tabla 4), como medidas recomendadas en la literatura de acuerdo a nuestro tamaño muestral. La Chi-cuadrado normalizada se encuentra entre 1 (Hair et al. 1999) y 5 (Jöreskog y Sörbom 1986), los estadísticos GFI y AGFI superan al valor mínimo recomendado de 0,90 (Bollen y Long 1993), CFI se encuentra muy próximo a 1 (Bentler 1990); mientras que el RMSEA se sitúa muy por debajo del límite máximo de referencia de 0,08 (Hair et al. Íbid.). Comparando los índices de ajuste con sus correspondientes valores recomendados se deduce un buen ajuste del modelo, aprobando la especificación hecha al modelo.

Las hipótesis planteadas en este estudio tienen la intención de probar la relación entre el conocimiento que tienen los individuos del medio ambiente y sus valores personales sobre su intención conductual. También la influencia que la intención de conducta tiene sobre el comportamiento ecológico. De la revisión de los coeficientes estandarizados, todos ellos presentan el signo esperado y son significativos; por tanto, las tres hipótesis causales propuestas son aceptadas (Tabla 4). En consecuencia, el conocimiento y los valores son antecedentes de la intención de conducta $(\mathrm{H} 1$ y H2); y esta, a su vez, influye directamente en el comportamiento ecológico (H3).

\section{Conclusiones}

Los problemas ambientales han propiciado un cambio en la sociedad, que se ha traducido en una modificación en los hábitos de vida y consumo de los individuos hacia comportamientos ecológicos y respetuosos con el medio ambiente. Bajo este contexto, este trabajo persigue un doble objetivo. Por una parte, avanzar en el conocimiento de las razones que hacen que un individuo se comporte de un modo ecológico identificando los factores 0 variables que motivan este comportamiento. $Y$ por otra, validar a nivel confirmatorio los instrumentos de medida del comportamiento ecológico y las variables que le anteceden.

En relación con el primer objetivo, formulamos un modelo estructural, basado en la teoría de la acción razonada (Fishbein y Ajzen 1975) y el enfoque tridimensional de la actitud capaz de explicar, a través del conocimiento de los individuos, sus valores e intenciones, el $87 \%$ del comportamiento que un individuo desarrolla con el fin de preservar o conservar 0 , al menos, no perjudicar al medio ambiente. Hemos comprobado que tanto el conocimiento como los valores son susceptibles de generar de forma indirecta actuaciones ecológicas, mediadas por las intenciones. También que las intenciones ejercen una influencia directa sobre la conducta. Según el efecto que producen, la variable intenciones es la más importante, seguida del conocimiento y, en último lugar y 
con una influencia similar, los valores. Nuestros resultados están en línea con los alcanzados por autores como Kaiser et al. (1999), Fraj y Martínez (2005) o, más recientemente, Aguilar-Luzón et al. (2012), aunque el porcentaje de varianza explicada del comportamiento ecológico en estos estudios es mucho menor. En el estudio de Kaiser et al. (1999), los autores, utilizando los 3 componentes de la actitud lograron explicar un $38 \%$ del comportamiento ecológico en una muestra de más de 1000 individuos en Suiza. En la misma línea, Fraj y Martínez (2005) explicaron el $24 \%$ de la varianza del comportamiento mediante una muestra de 573 individuos de la ciudad de Zaragoza. Por su parte, Aguilar-Luzón et al. (2012) consiguieron explicar un $37 \%$ del comportamiento específico de separación de vidrio en una muestra de 154 amas de casa españolas.

En cuanto al segundo objetivo, un conjunto de escalas específicas existentes en la literatura se han traducido, adaptado y analizado respecto a sus características psicométricas con el fin de ser utilizadas en estudios posteriores. De este modo, se ha demostrado empíricamente que la escala conocimiento viene determinada por dos factores. El primero se refiere al conocimiento que el individuo tiene respecto a las consecuencias del cambio climático, y el segundo, sobre el conocimiento de los efectos de los metales tóxicos, especialmente dañinos para el metabolismo humano. La escala sobre los valores queda compuesta por una única dimensión formada por 5 ítems que reflejan los principios de los individuos hacia el medio ambiente y el bienestar de otras personas. El constructo intención de comportamiento se estructura en una única dimensión formada por dos indicadores que reflejan la preferencia de los individuos con relación al uso del automóvil. Finalmente, el instrumento de medida comportamiento ecológico se establece como de segundo orden y se compone de dos dimensiones, la gestión ecológica de basuras y la implicación personal con otros en acciones de protección de la naturaleza. Hasta donde podemos saber, no existen evidencias empíricas de carácter confirmatorio de las escalas de medición de las variables utilizadas, para el contexto español.

\section{IMPLICACIONES PRÁCTICAS}

Nuestros resultados parecen indicar que la intención de una persona para actuar de manera responsable con el medio ambiente es esencial para que se comporte ecológicamente. También, que buena parte de las intenciones se basan en el conocimiento sobre los problemas ambientales que un individuo ha adquirido a través de su tradición cultural, la difusión en los medios de comunicación de conocimientos científicos y su propia experiencia (Barreiro et al. 2002), así como los valores 0 principios que guían a las personas y representan su preocupación por el entorno físico y la biosfera, su preocupación por el bienestar de otras personas y la preocupación por uno mismo (Stern et al. 1993).

Con ello, los gestores de empresas privadas y responsables de instituciones públicas, conociendo los elementos sobre los que el usuario define su comportamiento ecológico, tanto en lo relativo a su implicación medioambiental como a la gestión ecológica de basuras, deberían incluir en su planificación cuestiones que actúen sobre el conocimiento de los individuos sobre el medio ambiente, que orienten los valores que guían la vida de las personas y sus intenciones, para que estas se comporten de un modo más ecológico y responsable.

De este modo, sugerimos a los organismos públicos 1) el desarrollo de campañas de comunicación gubernamentales cognitivas o que avancen en el conocimiento básico y experto de los individuos sobre los problemas ecológicos y la forma de proteger el medio ambiente; 2) el fomento de determinadas conductas o desmotivación de otras, como puede ser, la utilización de medios de transporte públicos o la conducción responsable; 3 ) la promoción de iniciativas de voluntariado o de integración con la naturaleza, por ejemplo, en todos los niveles educativos, y especialmente en las escuelas.

Respecto a las empresas privadas, estas pueden actuar en el comportamiento ecológico de las personas aportando información y formación a la población no sólo sobre los problemas ambientales y las ventajas de proteger el medio ambiente sino también acerca de «las características, ventajas y beneficios ambientales asociados al producto y su impacto real en la preservación ecológica» (Akehurst et al. 2012: 984); por ejemplo, aportando al consumidor «consejos sobre cómo utilizar el producto más eficientemente, y consejos sobre la reutilización, reparación, reciclaje y desecho de ese producto» (Welford 1995, citado en Raufflet, Lozano, Barrera y García 2012: 151). También podrían utilizarse campañas de comunicación sectoriales 0 individuales informativas y de concienciación ambiental a través de los medios en los que los ciudadanos confían más, esto es, la televisión, los científicos y las asociaciones medioambientales (Ministerio de Medio Ambiente, Medio Rural y Marino de España 2011).

Por último, proponemos la promoción de iniciativas de colaboración con una causa de índole ambiental y/o social, ya que estas son capaces de actuar sobre el conocimiento, la concienciación, los valores, las intenciones y la propia conducta responsable del individuo (Kotler y Keller 2012).

\section{LIMITACIONES Y FUTURAS LÍNEAS DE INVESTIGACIÓN}

Aunque creemos que las variables utilizadas son suficientes para representar el comportamiento ecológico 
(explican un $87 \%$ de la varianza del constructo), el modelo puede ser mejorado incluyendo otras variables. En consecuencia, para futuras investigaciones sería interesante la incorporación de variables fuera del control de los individuos y no solo variables internas, ya que la realización de una conducta ecológica no solo viene determinada por éstas, sino también por la existencia de factores externos o contextuales y grupales que serán los que faciliten o inhiban la realización de las conductas ambientales (Hernández y Suárez 2006; Aguilar etal. 2005). Entre estos, destacamos los costes situacionales, esto es, la dificultad para realizar una conducta ecológica (Kaiser y Schultz 2009), la proximidad a los centros de reciclaje o la confianza en la actuación de terceros tales como gobiernos, empresas u organismos medioambientales (Fraj y Martínez 2005).

\section{REFERENCIAS BibLIOGRÁfICAS}

Aguilar, M. C., A. Monteoliva y J. M. García. 2005. «Influencia de las normas, los valores, las creencias proambientales y la conducta pasada sobre la intención de reciclar». Medio Ambiente y Comportamiento Humano 6:23-36.

Aguilar-Luzón, M. C., J. M. García-Martínez, A. Calvo-Salgueiro y J. M. Salinas. 2012. "Comparative study between the theory of planned behavior and value-belief-norm model regarding the environment, on Spanish housewives' recycling behavior». Journal of Applied Social Psychology 42:2797-2833. http:/l dx.doi.org/10.1111/j.1559-1816.2012.00962.x

Ajzen, I. 1991. "The theory of planned behavior». Organizational Behavior and Human Decision Processes 50:179-211. http:// dx.doi.org/10.1016/0749-5978(91)90020-T

Akehurst, G., C. Afonso y H. M. Gonçalves. 2012. «Re-examinig green purchase behavior and the green consumer profile: new evidences». Management Decision 50:972-988. http://dx.doi.org/10.1108/00251741211227726

Amérigo, M. 2006. «La investigación en España sobre actitudes proambientales y comportamiento ecológico». Medio Ambiente y Comportamiento Humano 7:45-71.

Anderson, J. C. y D. W. Gerbing. 1988. «Structural equation modeling in practice: a review and recommended two-step approach?». Psychological Bulletin 103:411-23. http://dx.doi. org/10.1037/0033-2909.103.3.411

Bagozzi, R. P., Y. Yi y L. W. Phillips. 1991. «Assessing construct validity in organizational research». Administrative Science Quarterly 36:421-458. http://dx.doi.org/10.2307/2393203

Bamberg, S. y G. Möser. 2007. "Twenty years after Hines, Hungerford and Tomera: a new meta-analysis of psychosocial determinants of pro-environmental behavior». Journal of Environmental Psychology 27:14-25. http:// dx.doi.org/10.1016/j.jenvp.2006.12.002

Barreiro, J. M., M. A. López, F. Losada y E. Ruzo. 2002. «Análisis de las dimensiones cognoscitiva y afectiva del comportamiento ecológico del consumidor». Revista Galega de Economía 11:1-21.

Bentler, P. M. 1990. "Comparative fit indices in structural models». Psychological Bulletin 107:238-246. http://dx.doi.org/10.1037/0033-2909.107.2.238 PMid:2320703

Bollen, K., y J. Long. 1993. Testing structural equation models. Newbury Park, CA: Sage Publications.
Bollen, K. A. 1989. Structural equations with latent variables. New York: John Wiley \& Sons. http://dx.doi.org/10.1002/9781118619179 PMCid:PMC1133599

Brosdahl, D. J. C. y J. M. Carpenter. 2010. «Consumer knowledge of the environmental impacts of textile and apparel production. Concern for the environment, and environmentally friendly consumption behavior». Journal of Textile and Apparel, Technology and Management 6:1-9.

Calvo, A., M. C. Aguilar y M. P. Berrios. 2008. «El comportamiento ecológico responsable: un análisis desde los valores biosféricos, sociales-altruistas y egoístas». Revista Electrónica de Investigación y Docencia (REID) 1:11-25.

Castro, R. 2002. «¿Estamos dispuestos a proteger nuestro ambiente? Intención de conducta y comportamiento proambiental». Medio Ambiente y Comportamiento Humano 3:107-118.

Churchill, G. A. 1979. «A Paradigm for developing better measures of marketing constructs». Journal of Marketing Research 16:64-73. http://dx.doi.org/10.2307/3150876

Do Paço, A. y M. Raposo. 2009. «Green segmentation: an application to the Portuguese consumer market». Marketing Intelligence and Planning 27:364-379. http://dx.doi. org/10.1108/02634500910955245

Esteban, A., J. A. Mondéjar y M. Cordente. 2009. «Algunos conceptos de marketing y medio ambiente: una propuesta de revisión». Revista de Economía, Sociedad, Turismo y Medio Ambiente 8-9:47-75.

Eurobarómetro. 2011. Directorate-General for the Environment / TNS Opinion \& Social. Special eurobarometer 365: Attitudes of European citizens towards the environment. Bruselas: Directorate-General Communication- TNS Opinion \& Social.

Fishbein, M. and I. Ajzen. 1975. Belief, attitude, intention, and behavior: An introduction to theory and research. Reading, MA: Addison-Wesley.

Fornell, C. y D. F. Larcker. 1981. «Evaluating structural equation models with unobservable variables and measurement error». Journal of Marketing Research 18:39-50. http://dx.doi. org/10.2307/3150980 http://dx.doi.org/10.2307/3151312

Fraj, E. y E. Martínez. 2004. «El consumo ecológico explicado a través de los valores y estilos de vida. Implicaciones de la estrategia medioambiental de la empresa». Cuadernos de CC.EE y EE 46:33-54.

Fraj, E. y E. Martínez. 2005. «El nivel de conocimiento medioambiental como factor moderador de la relación entre la actitud y el comportamiento ecológico». Investigaciones Europeas de Dirección y Economía de la Empresa 11:223-243.

Fraj, E. y E. Martínez. 2006. «Ecological consumer behaviour: an empirical analysis». International Journal of Consumer Studies 26-33.

Fraj, E., I. Grande y E. M. Martínez. 2002. "Las variables de actitud y de conocimiento como determinantes del comportamiento ecológico». Investigación y Marketing 74:56-66.

Fraj, E., E. Martínez e I. Grande. 2004. «Un estudio exploratorio sobre las variables psicográficas que influyen en el comportamiento del consumidor ecológico». Revista de Economía y Empresa 50:61-87.

Frick, J., F. Kaiser y M. Wilson. 2004. «Environmental knowledge and conservation behavior: exploring prevalence and structure in a representative sample». Personality and Individual Differences 37:1597-1613. http://dx.doi.org/10.1016/j.paid.2004.02.015

González, A. 2002. La preocupación por la calidad del medio ambiente. Un modelo cognitivo sobre la conducta ecológica. Memoria presentada para optar al grado de Doctor. Departamento de psicología social, Universidad Complutense de Madrid.

González, A. y M. Amérigo. 1998. «La preocupación ambiental como función de valores y creencias». Revista de Psicología Social 13:453-461. http://dx.doi.org/10.1174/021347498760349706 
González, A. y M. Amérigo. 1999. «Actitudes hacia el medio ambiente y conducta ecológica». Psicothema 11:13-25.

Hair, J. F., R. E. Anderson, R. L. Tatham y W. C. Black. 1999. Análisis multivariante. Madrid: Prentice Hall. PMCid:PMC2326768

Hines, J. M., H. R. Hungerford y A. N. Tomera. 1987. «Analysis and synthesis of research on responsible environmental behavior: a metaanalysis». Journal of Environmental Education 18:1-18. http://dx.doi.org/10.1080/00958964.1987.9943482

Hu, L. T. y P. M. Bentler. 1999. "Cutoff criteria for fit indexes in covariance structure analysis: Conventional criteria versus new alternatives». Structural Equation Modeling 6:1-55. http:/l dx.doi.org/10.1080/10705519909540118

INE (Base de datos). 2011. Proyecciones de población a corto plazo. 2011-2021. Población residente en España a 1 de enero, por sexo, edad y año. Madrid: Instituto Nacional de Estadística.

Izagirre-Olaizola, J., A. Fernández-Sainz y M. A. Vicente-Molina. 2013. Antecedentes y barreras a la compra de productos ecológicos. Universia Business Review Segundo Trimestre: 108-127.

Jiménez, M. y R. Lafuente. 2010. «Defining and measuring environmental consciousness». Revista Internacional de Sociología 68:731-755. http://dx.doi.org/10.3989/ris.2008.11.03

Jöreskog, K. G. y D. Sörbom. 1986. LISREL VI: Analysis of linear structural relationships by maximum likelihood, instrumental variables, and least squares methods. Mooresville, IN: Scientific Software.

Kaiser, F. y P. W. Schultz. 2009. "The attitude-behavior relationship: A test of three models of the moderating role of behavioral difficulty». Journal of Applied Social Psychology 39:186-207. http://dx.doi.org/10.1111/j.1559-1816.2008.00435.x

Kaiser, F. G. 1998. «A general measure of ecological behavior». Journal of Applied Social Psychology 28:395-422. http://dx.doi.org/10.1111/j.1559-1816.1998.tb01712.x

Kaiser, F. G., S. Wölfing y U. Fuhrer. 1999. «Environmental attitude and ecological behavior». Journal of Environmental Psychology 19:1-19. http://dx.doi.org/10.1006/jevp.1998.0107

Kaiser, F. G. y U. Fuhrer. 2003. «Ecological behavior's dependency on different forms of knowledge». Applied Psychology: An International Review 52:598-613. http://dx.doi.org/10.1111/1464-0597.00153

Karp, D. 1996. "Values and their effect on pro-environmental behavior». Environment and Behavior 28:111-133. http://dx.doi. org/10.1177/0013916596281006

Kotler, P. y K. Keller. 2012. Dirección de Marketing, 14 ed. Madrid: Pearson Educación.

Lévy, J. P. y J. Varela. 2003. Análisis multivariable para las ciencias sociales. Madrid: Pearson Educación.

López, J. y J. García. 2007. "Valores, actitudes y comportamiento ecológico modelados con una red bayesiana». Medio Ambiente y Comportamiento Humano 8:159-175.

Luque, T. 2000. Técnicas de análisis de datos en investigación de mercados. Madrid: Pirámide.

Luzio, J. P. P. y F. Lemke. 2013. «Exploring green consumers' product demands and consumption processes: The case of Portuguese green consumers». European Business Review 25:281-300. http://dx.doi.org/10.1108/09555341311314825

Ministerio de Medio Ambiente, Medio Rural y Marino. 2011. Percepción social del Medio Ambiente. Análisis y Prospectiva - Serie Medio Ambiente $n^{0} 5$. Subdirección General de Análisis, Prospectiva y Coordinación, Subsecretaría. Ministerio de Medio Ambiente y Medio Rural y Marino. NIPO: 770-11006-4.

Milfont, T. L. y J. Duckitt. 2004. «The structure of environmental attitudes: A first- and second- order confirmatory factor analysis». Journal of Environmental Psychology 24:289-303. http://dx.doi.org/10.1016/j.jenvp.2004.09.001

Moreno, M., J. A. Corraliza y J. P. Ruiz. 2005. «Escala de actitudes ambientales hacia problemas específicos». Psicothema 17:502-506.
Numally, J. C. 1978. «Theory of measurement error». En Psychometric theory (2nd ed. New York: McGraw Hill. En Lévy y Varela (2003. Raufflet, E., J. F. Lozano, E. Barrera y C. García. 2012. Responsabilidad social empresarial. Pearson Educación. México.

Peattie, K. 2001. «Golden goose or wild goose? The hunt for the green consumer». Business Strategy and the Environment 10:187199. http://dx.doi.org/10.1002/bse.292

Roberts, J. A. y D. R. Bacon. 1997. «Exploring the subtle relationship between environmental concern and ecologically conscious consumer behavior». Journal of Business Research 40:7989. http://dx.doi.org/10.1016/S0148-2963(96)00280-9

Schiffman, L. G., L. L. Kanuk y H. Hansen. 2008. Consumer behavior. A European outlook. Pearson Education, New Jersey.

Schwartz, S. H. 1992. "Universals in the content and structure of values, theoretical advances and empirical test in 20 countries». Advances in Experimental Social Psychology 10:221-279. http://dx.doi.org/10.1016/s0065-2601(08)60281-6

Stern, P. C. y T. Dietz. 1994. "The value basis of environmental concern». Journal of Social Issues 50:65-84. http://dx.doi. org/10.1111/j.1540-4560.1994.tb02420.x

Stern, P. C., T. Dietz y L. Kalof. 1993. «Value orientations, gender and environmental concern». Environment and Behavior 25:322348. http://dx.doi.org/10.1177/0013916593255002

Stern, P. C., Dietz, T., Kalof, L. y Guagnano, G. A. 1995. «Values, beliefs and proenvironmental action: Attitude formation toward emergent attitude objects». Journal of Applied Social Psychology 25:1611-1636. http://dx.doi.org/10.1111/j.1559-1816.1995. tb02636.x

(Del) Val, A. 2011. «El problema de los residuos en la sociedad del bienestar». Boletín CF+S 50:29-38. Consultado (http://habitat. aq.upm.es/boletin/n50/aaval.html). (Consulta: 07/11/2012).

Vicente, M. A. y C. Aldamiz-Echevarría. 2003. «Aproximación al perfil sociodemográfico del consumidor ecológico a través de la evidencia empírica: propuestas para el desarrollo del mix de marketing». Boletín Económico de ICE 2777:25-39.

Vozmediano, L. y C. San Juan. 2005. «Escala nuevo paradigma ecológico: propiedades psicométricas con una muestra española obtenida a través de Internet». Medio Ambiente y Comportamiento Humano 6:37-49.

Willett, J. B. yA. G. Sayer. 1994. «Using covariance structure analysis to detect correlates and predictors of change». Psychological Bulletin 116:363-381. http://dx.doi.org/10.1037/0033-2909.116.2.363

Young, W., K. Hwang, S. Mcdonald y C. J. Oates. 2010. «Sustainable consumption: green consumer behaviour when purchasing products». Sustainable Development 18:20-31.

MARÍA JESÚS LÓPEZ MIGUENS. Es Doctora por la Universidad de Vigo y Licenciada en Administración y Dirección de Empresas. Su docencia se concentra en el área de Marketing. Es autora de diversos artículos en revistas científicas y de ponencias en congresos a nivel nacional e internacional.

PAULA ÁLVAREZ GONZÁLEZ. Licenciada en Administración y Dirección de Empresas por la Universidad de Vigo. Autora de artículos en revistas científicas y ponencias en congresos a nivel nacional e internacional.

ENCARNACIÓN GONZÁLEZ VÁZQUEZ. Catedrática de Universidad del área de Comercialización e Investigación de Mercados en la Universidad de Vigo. Doctora en Ciencias Económicas y Empresariales. Autora de diversas publicaciones en revistas nacionales e internacionales, de capítulos en diferentes libros y de ponencias en congresos. Investigadora principal en diversos proyectos de investigación financiados por Administraciones Públicas. 